\title{
Controlled Quantum Teleportation via Four Particle Asymmetric Entangled State *
}

\author{
Pan-ru Zhao ${ }^{1}$, Hui $\mathrm{Xu}^{1}$, Yuan-hong Tao ${ }^{1 \dagger}$ \\ Department of Mathematics, College of Science, Yanbian University, Yanji, Jilin 13302 \\ _This work is supported by Natural Science Foundation of China under number 11361065. \\ †Corresponding author: Yuan-hong Tao, E-mail: taoyuanhong12@126.com
}

\begin{abstract}
A general scheme for controlled teleportation of an arbitrary single-qubit state with four-qubit asymmetric state is proposed. In this scheme, the sender performs Bell measurement on his particle, the two controllers and the receiver perform joint unitary operation on the rest particles. Finally, the receiver can reconstruct the single particle by introducing an auxiliary particle, he first does unitary transformation on his particle and the auxiliary particle, then performs a Von Neumann measurement on the auxiliary particle. Thus the scheme can be realized in certain probability.
\end{abstract}

Keywords: controlled quantum teleportation; asymmetric entangled state; Bell measurement; Von Neumann measurement

\section{Introduction}

The first teleportation scheme of one-particle unknown state via an Einstein-Podolsky-Rosen pair with the help of some classical information is proposed by Bennett et al[1] in 1993. Up to now, there are many achievements in theory and experiment about teleportation, which can be seen in the literature [1 _ 12] and their literature. The key to quantum teleportation is to establish a quantum channel between the sender and the receiver. There are many choices of quantum channels, including two-particle channels[2_4], three-particle channels[5_7], four-particle channels[8_10], six-particle channels[11] and nparticle channel[12]. In 2016, Zhang Jianzhong et al[10] proposed a scheme of two quantum secret sharing scheme based on four-qubit entangled ststes. From [10], quantum teleportation based on asymmetric quantum channel can transmit more information than the quantum teleportation of symmetric quantum channel. In this paper, we intend to discuss a scheme of controlled quantum teleportation, the state we want to teleport is single-qubit state, the channel we choose is four particle asymmetric entangled state. There includes four parties, one sender, one receiver, two controllers. We will present its whole process according to three cases and we will calculate all successful probabilities of the teleportatiion.

\section{Controlled Quantum Teleportation Scheme}

A one-particle unknown state to be teleported is as follows

$$
|\psi\rangle_{0}=(\alpha|0\rangle+\beta|1\rangle)_{0}
$$

where $\alpha, \beta$ is complex, and $|\alpha|^{2}+|\beta|^{2}=1$.

In our scheme, there are four parties, Alice, Bob, Charlie and David, supposing that they each have a particle, denoting 1, 2, 3, 4 respectively. The state of the four state is as follows

$$
|\psi\rangle_{1234}=(a|0011\rangle+b|1101\rangle+b|1010\rangle)_{1234}
$$

where $a, b$ is real, $a^{2}+2 b^{2}=1$ and $a^{2} \leq 2 b^{2}$.

The entire system which includes the unknown state 0 and quantum channel is denoted as follows

$$
|\zeta\rangle_{01234}=(\alpha|0\rangle+\beta|1\rangle)_{0} \otimes(a|0011\rangle+b|1101\rangle+b|1010\rangle)_{1234}
$$

In order to teleport state $|\psi\rangle_{0}$, Alice performs Bell measurement on particle 0 and 1 . Then the state $|\zeta\rangle_{01234}$ becomes any one of the following states

$$
\begin{aligned}
& \left|\eta_{1}\right\rangle={ }_{01}\left\langle\phi^{+} \mid \zeta\right\rangle_{01234}=\frac{1}{\sqrt{2}}(\alpha a|001\rangle+\beta b|101\rangle+\beta b|010\rangle)_{234} \\
& \left|\eta_{2}\right\rangle={ }_{01}\left\langle\phi^{-} \mid \zeta\right\rangle_{01234}=\frac{1}{\sqrt{2}}(\alpha a|001\rangle-\beta b|101\rangle-\beta b|010\rangle)_{234} \\
& \left|\eta_{3}\right\rangle={ }_{01}\left\langle\psi^{+} \mid \zeta\right\rangle_{01234}=\frac{1}{\sqrt{2}}(\alpha b|101\rangle+\alpha b|010\rangle+\beta a|011\rangle)_{234}
\end{aligned}
$$


where $\left|\phi^{ \pm}\right\rangle_{01}=\frac{1}{\sqrt{2}}(|00\rangle \pm|11\rangle)_{01},\left|\psi^{ \pm}\right\rangle_{01}=\frac{1}{\sqrt{2}}(|01\rangle \pm|10\rangle)_{01}$.

Among Alice, Bob, Charlie and David, there are one sender, one receiver, and two controllers. We suppose that Alice is the sender, any one of Bob, Charlie and David is the receiver, and the rest two are the controllers.

According to the difference of the receiver and the controllers, we analyze the scheme in the following three cases.

Case 1. David is the receiver, Bob and Charlie are the controllers.

Based on the above discussion, if Bob and Charlie agree to help David, then they three perform the following joint unitary transformation on particle 2,3,4.

$$
\begin{gathered}
\Omega_{D}=|000\rangle\langle 000|+| 001\rangle\langle 001|+| 011\rangle\langle 011|+| 100\rangle\langle 100|+| 110\rangle\langle 110|+| 111\rangle\langle 111| \\
+\frac{1}{\sqrt{2}}(|010\rangle\langle 010|+| 010\rangle\langle 101|+| 101\rangle\langle 010|-| 101\rangle\langle 101|)
\end{gathered}
$$

It's matrix is

$$
\Omega_{D}=\left(\begin{array}{cccccccc}
1 & 0 & 0 & 0 & 0 & 0 & 0 & 0 \\
0 & 1 & 0 & 0 & 0 & 0 & 0 & 0 \\
0 & 0 & \frac{1}{\sqrt{2}} & 0 & 0 & \frac{1}{\sqrt{2}} & 0 & 0 \\
0 & 0 & 0 & 1 & 0 & 0 & 0 & 0 \\
0 & 0 & 0 & 0 & 1 & 0 & 0 & 0 \\
0 & 0 & \frac{1}{\sqrt{2}} & 0 & 0 & -\frac{1}{\sqrt{2}} & 0 & 0 \\
0 & 0 & 0 & 0 & 0 & 0 & 1 & 0 \\
0 & 0 & 0 & 0 & 0 & 0 & 0 & 1
\end{array}\right)
$$

Then the four states $\left|\eta_{1}\right\rangle,\left|\eta_{2}\right\rangle,\left|\eta_{3}\right\rangle,\left|\eta_{4}\right\rangle$ become the following states

$$
\begin{aligned}
& \left|\zeta_{1}\right\rangle=\left(\frac{1}{\sqrt{2}} \alpha a|011\rangle+\beta b|010\rangle\right)_{234}=\frac{1}{\sqrt{2}}|01\rangle_{23}(\alpha a|1\rangle+\sqrt{2} \beta b|0\rangle)_{4} \\
& \left|\zeta_{2}\right\rangle=\left(\frac{1}{\sqrt{2}} \alpha a|011\rangle-\beta b|010\rangle\right)_{234}=\frac{1}{\sqrt{2}}|01\rangle_{23}(\alpha a|1\rangle-\sqrt{2} \beta b|0\rangle)_{4} \\
& \left|\zeta_{3}\right\rangle=\left(\alpha b|010\rangle+\frac{1}{\sqrt{2}} \beta a|011\rangle\right)_{234}=\frac{1}{\sqrt{2}}|01\rangle_{23}(\sqrt{2} \alpha b|0\rangle+\beta a|1\rangle)_{4} \\
& \left|\zeta_{4}\right\rangle=\left(\alpha b|010\rangle-\frac{1}{\sqrt{2}} \beta a|011\rangle\right)_{234}=\frac{1}{\sqrt{2}}|01\rangle_{23}(\sqrt{2} \alpha b|0\rangle-\beta a|1\rangle)_{4}
\end{aligned}
$$

Now the states of the particles 4 can be denoted as follows, respectively

$$
\left|\varphi_{1}\right\rangle=\frac{1}{\sqrt{2}}(\alpha a|1\rangle+\sqrt{2} \beta b|0\rangle)_{4}
$$




$$
\begin{aligned}
& \left|\varphi_{2}\right\rangle=\frac{1}{\sqrt{2}}(\alpha a|1\rangle-\sqrt{2} \beta b|0\rangle)_{4} \\
& \left|\varphi_{3}\right\rangle=\frac{1}{\sqrt{2}}(\sqrt{2} \alpha b|0\rangle+\beta a|1\rangle)_{4} \\
& \left|\varphi_{4}\right\rangle=\frac{1}{\sqrt{2}}(\sqrt{2} \alpha b|0\rangle-\beta a|1\rangle)_{4}
\end{aligned}
$$

From the above forms of particle 4, David can not yet get the unknown particles by unitary transformations. So he has to introduce an auxiliary particle $|0\rangle_{f}$. He first performs a unitary transformation on particle 4 and the auxiliary particle, then he performs a Von Neumann measurement on the auxiliary particle. Thus he reconstruct the unknown particles successfully with a certain probability.

If the state of the particle 4 is $\left|\varphi_{1}\right\rangle$ or $\left|\varphi_{2}\right\rangle$, David performs the following unitary measurement $U_{1}$ on particle 4 and the auxiliary particle

$$
U_{1}=|00\rangle\langle 10|-| 01\rangle\left\langle 11\left| \pm \frac{a}{\sqrt{2} b}\right| 10\right\rangle\left\langle 00\left|+\sqrt{1-\frac{a^{2}}{2 b^{2}}}\right| 10\right\rangle\left\langle 01\left|+\sqrt{1-\frac{a^{2}}{2 b^{2}}}\right| 11\right\rangle\left\langle 00\left|\mp \frac{a}{\sqrt{2} b}\right| 11\right\rangle\langle 01|
$$

It's matrix is

$$
U_{1}=\left(\begin{array}{cccc}
0 & 0 & 1 & 0 \\
0 & 0 & 0 & -1 \\
\pm \frac{a}{\sqrt{2} b} & \sqrt{1-\frac{a^{2}}{2 b^{2}}} & 0 & 0 \\
\sqrt{1-\frac{a^{2}}{2 b^{2}}} & \mp \frac{a}{\sqrt{2 b}} & 0 & 0
\end{array}\right)
$$

Hence the state of particle 4 and the auxiliary particle become the following state

$$
U_{1} \frac{1}{\sqrt{2}}(\alpha a|1\rangle \pm \sqrt{2} \beta b|0\rangle)_{4}|0\rangle_{f}=\frac{1}{\sqrt{2}}\left[a(\alpha|0\rangle+\beta|1\rangle)_{4}|0\rangle_{f} \pm \sqrt{2} \beta b \sqrt{1-\frac{a^{2}}{2 b^{2}}}|1\rangle_{4}|1\rangle_{f}\right]
$$

Then, David performs a Von Neumann measurement on the auxiliary particle. From the above equation, we can see that if the measurement result of the auxiliary particle is $|1\rangle_{f}$, the teleportation fails. If the result is $|0\rangle_{f}$, the teleportation is succeed with probability $\frac{a^{2}}{2}$.

If the state of the particle 4 is $\left|\varphi_{3}\right\rangle$ or $\left|\varphi_{4}\right\rangle$, David performs the following unitary measurement $V_{1}$ on particle 4 and the auxiliary particle

$$
V_{1}=\frac{a}{\sqrt{2} b}|00\rangle\left\langle 00\left|+\sqrt{1-\frac{a^{2}}{2 b^{2}}}\right| 00\right\rangle\left\langle 01\left|+\sqrt{1-\frac{a^{2}}{2 b^{2}}}\right| 01\right\rangle\left\langle 00\left|-\frac{a}{\sqrt{2} b}\right| 01\right\rangle\langle 01| \pm| 10\rangle\langle 10|\mp| 11\rangle\langle 11|
$$

It's matrix is

$$
V_{1}=\left(\begin{array}{cccc}
\frac{a}{\sqrt{2} b} & \sqrt{1-\frac{a^{2}}{2 b^{2}}} & 0 & 0 \\
\sqrt{1-\frac{a^{2}}{2 b^{2}}} & -\frac{a}{\sqrt{2} b} & 0 & 0 \\
0 & 0 & \pm 1 & 0 \\
0 & 0 & 0 & \mp 1
\end{array}\right)
$$

Hence the state of particle 4 and auxiliary particle $|0\rangle_{f}$ become the following state 


$$
V_{1} \frac{1}{\sqrt{2}}(\sqrt{2} \alpha b|0\rangle \pm \beta a|1\rangle)_{4}|0\rangle_{f}=\frac{1}{\sqrt{2}}\left[a(\alpha|0\rangle+\beta|1\rangle)_{4}|0\rangle_{f}+\sqrt{2} \alpha b \sqrt{1-\frac{a^{2}}{2 b^{2}}}|0\rangle_{4}|1\rangle_{f}\right]
$$

Then, David perform a Von Neumann measurement on the auxiliary particle. From the above equation, we can see that if the measurement result of the auxiliary particle is $|1\rangle_{f}$, the teleportation fails. If the result is $|0\rangle_{f}$, the teleportation is succeed with probability $\frac{a^{2}}{2}$.

So the total probability of the successful teleportation is $\frac{a^{2}}{2} \times 4=2 a^{2}$.

Case 2. Bob is the receiver, David and Charlie are the controllers.

Based on the above discussion, if David and Charlie agree to help Bob, then they three perform the following joint unitary transformation on particle $2,3,4$

$$
\begin{gathered}
\Omega_{B}=|000\rangle\langle 000|+| 001\rangle\langle 001|+| 011\rangle\langle 011|+| 100\rangle\langle 100|+| 110\rangle\langle 110|+| 111\rangle\langle 111| \\
+\frac{1}{\sqrt{2}}(-|010\rangle\langle 010|+| 010\rangle\langle 101|+| 101\rangle\langle 010|+| 101\rangle\langle 101|)
\end{gathered}
$$

Then the four states $\left|\eta_{1}\right\rangle,\left|\eta_{2}\right\rangle,\left|\eta_{3}\right\rangle,\left|\eta_{4}\right\rangle$ become the following states

$$
\begin{aligned}
& \left|\zeta_{1}^{\prime}\right\rangle=\left(\frac{1}{\sqrt{2}} \alpha a|011\rangle+\beta b|101\rangle\right)_{234}=\frac{1}{\sqrt{2}}(\alpha a|01\rangle+\sqrt{2} \beta b|10\rangle)_{23}|1\rangle_{4} \\
& \left|\zeta_{2}^{\prime}\right\rangle=\left(\frac{1}{\sqrt{2}} \alpha a|011\rangle-\beta b|101\rangle\right)_{234}=\frac{1}{\sqrt{2}}(\alpha a|01\rangle-\sqrt{2} \beta b|10\rangle)_{23}|1\rangle_{4} \\
& \left|\zeta_{3}^{\prime}\right\rangle=\left(\alpha b|101\rangle+\frac{1}{\sqrt{2}} \beta a|011\rangle\right)_{234}=\frac{1}{\sqrt{2}}(\sqrt{2} \alpha b|10\rangle+\beta a|01\rangle)_{23}|1\rangle_{4} \\
& \left|\zeta_{4}^{\prime}\right\rangle=\left(\alpha b|101\rangle-\frac{1}{\sqrt{2}} \beta a|011\rangle\right)_{234}=\frac{1}{\sqrt{2}}(\sqrt{2} \alpha b|10\rangle-\beta a|01\rangle)_{23}|1\rangle_{4}
\end{aligned}
$$

Then, Bob takes particle 2 as the control bit and particle 3 as the target bit, $\left|\zeta_{1}^{\prime}\right\rangle,\left|\zeta_{2}^{\prime}\right\rangle,\left|\zeta_{3}^{\prime}\right\rangle,\left|\zeta_{4}^{\prime}\right\rangle$ do controlled non-gate operation, get

$$
\begin{aligned}
\left|\zeta_{1}^{\prime \prime}\right\rangle & =\frac{1}{\sqrt{2}}(\alpha a|0\rangle+\sqrt{2} \beta b|1\rangle)_{2}|11\rangle_{34} \\
\left|\zeta_{2}^{\prime \prime}\right\rangle & =\frac{1}{\sqrt{2}}(\alpha a|0\rangle-\sqrt{2} \beta b|1\rangle)_{2}|11\rangle_{34} \\
\left|\zeta_{3}^{\prime \prime}\right\rangle & =\frac{1}{\sqrt{2}}(\sqrt{2} \alpha b|1\rangle+\beta a|0\rangle)_{2}|11\rangle_{34} \\
\left|\zeta_{4}^{\prime \prime}\right\rangle & =\frac{1}{\sqrt{2}}(\sqrt{2} \alpha b|1\rangle-\beta a|0\rangle)_{2}|11\rangle_{34}
\end{aligned}
$$

Now the state of the particle 2 can be denoted as follows, respectively

$$
\begin{aligned}
& \left|\varphi_{1}^{\prime}\right\rangle=\frac{1}{\sqrt{2}}(\alpha a|0\rangle+\sqrt{2} \beta b|1\rangle)_{2} \\
& \left|\varphi_{2}^{\prime}\right\rangle=\frac{1}{\sqrt{2}}(\alpha a|0\rangle-\sqrt{2} \beta b|1\rangle)_{2} \\
& \left|\varphi_{3}^{\prime}\right\rangle=\frac{1}{\sqrt{2}}(\sqrt{2} \alpha b|1\rangle+\beta a|0\rangle)_{2} \\
& \left|\varphi_{4}^{\prime}\right\rangle=\frac{1}{\sqrt{2}}(\sqrt{2} \alpha b|1\rangle-\beta a|0\rangle)_{2}
\end{aligned}
$$


From the above forms of particle 2, Bob can not yet get the unknown particles by unitary transformations. So he has to introduce an auxiliary particle $|0\rangle_{z}$. He first performs a unitary transformation on particle 2 and auxiliary particle $|0\rangle_{z}$, then he performs a Von Neumann measurement on the auxiliary particle. Thus he reconstruct the unknown particles successfully with a certain probability.

If the state of the particle 2 is $\left|\varphi_{1}^{\prime}\right\rangle$ or $\left|\varphi_{2}^{\prime}\right\rangle$, Bob performs the following unitary measurement $U_{2}$ on particle 2 and the auxiliary particle

$$
U_{2}=|00\rangle\langle 00|-| 01\rangle\left\langle 01\left| \pm \frac{a}{\sqrt{2} b}\right| 10\right\rangle\left\langle 10\left|+\sqrt{1-\frac{a^{2}}{2 b^{2}}}\right| 10\right\rangle\left\langle 11\left|+\sqrt{1-\frac{a^{2}}{2 b^{2}}}\right| 11\right\rangle\left\langle 10\left|\mp \frac{a}{\sqrt{2} b}\right| 11\right\rangle\langle 11|
$$

Hence the state of particle 2 and auxiliary particle $|0\rangle_{z}$ become the following state

$$
U_{2} \frac{1}{\sqrt{2}}(\alpha a|0\rangle \pm \sqrt{2} \beta b|1\rangle)_{2}|0\rangle_{z}=\frac{1}{\sqrt{2}}\left[a(\alpha|0\rangle+\beta|1\rangle)_{2}|0\rangle_{z} \pm \sqrt{2} \beta b \sqrt{1-\frac{a^{2}}{2 b^{2}}}|1\rangle_{2}|1\rangle_{z}\right]
$$

Then, Bob performs a Von Neumann measurement on the auxiliary particle. From the above equation, we can see that if the measurement result of the auxiliary particle is $|1\rangle_{z}$, the teleportation fails. If the result is $|0\rangle_{z}$, the teleportation is succeed with probability $\frac{a^{2}}{2}$.

If the state of the particle 2 is $\left|\varphi_{3}^{\prime}\right\rangle$ or $\left|\varphi_{4}^{\prime}\right\rangle$, then Bob performs the following unitary measurement $V_{2}$ on particle 2 and the auxiliary particle

$$
V_{2}=-\sqrt{1-\frac{a^{2}}{2 b^{2}}}|00\rangle\left\langle 01\left|+\frac{a}{\sqrt{2} b}\right| 00\right\rangle\left\langle 10\left|+\frac{a}{\sqrt{2} b}\right| 01\right\rangle\left\langle 01\left|+\sqrt{1-\frac{a^{2}}{2 b^{2}}}\right| 01\right\rangle\langle 10| \pm| 10\rangle\langle 00|\mp| 11\rangle\langle 11|
$$

Hence the state of particle 2 and auxiliary particle $|0\rangle_{z}$ become the following state

$$
V_{2} \frac{1}{\sqrt{2}}(\sqrt{2} \alpha b|1\rangle \pm \beta a|0\rangle)_{2}|0\rangle_{z}=\frac{1}{\sqrt{2}}\left[a(\alpha|0\rangle+\beta|1\rangle)_{2}|0\rangle_{z}+\sqrt{2} \alpha b \sqrt{1-\frac{a^{2}}{2 b^{2}}}|0\rangle_{2}|1\rangle_{z}\right]
$$

Then, Bob performs a Von Neumann measurement on the auxiliary particle. From the above equation, we can see that if the measurement result of the auxiliary particle is $|1\rangle_{z}$, the teleportation fails. If the result is $|0\rangle_{z}$, the teleportation is succeed with probability $\frac{a^{2}}{2}$.

So the total probability of the successful teleportation is $\frac{a^{2}}{2} \times 4=2 a^{2}$.

Case 3. Charlie is the receiver, David and Bob are the controllers.

Similarly, based on the above discussion, if David and Bob agree to help Charlie. Firstly, they three perform the joint unitary transformation $\Omega_{B}$ on particle 2,3,4, then the state of particle 2, 3, 4 can be denoted as $\left|\zeta_{1}^{\prime}\right\rangle,\left|\zeta_{2}^{\prime}\right\rangle,\left|\zeta_{3}^{\prime}\right\rangle,\left|\zeta_{4}^{\prime}\right\rangle$. Secondly, Charlie takes particle 3 as the control bit and particle 2 as the target bit, the four state $\left|\zeta_{1}\right\rangle,\left|\zeta_{2}^{\prime}\right\rangle,\left|\zeta_{3}^{\prime}\right\rangle,\left|\zeta_{4}^{\prime}\right\rangle$ become the following state

$$
\begin{aligned}
\left|\zeta_{1}^{\prime \prime \prime}\right\rangle & =\frac{1}{\sqrt{2}}(\alpha a|1\rangle+\sqrt{2} \beta b|0\rangle)_{3}|11\rangle_{24} \\
\left|\zeta_{2}^{\prime \prime \prime}\right\rangle & =\frac{1}{\sqrt{2}}(\alpha a|1\rangle-\sqrt{2} \beta b|0\rangle)_{3}|11\rangle_{24} \\
\left|\zeta_{3}^{\prime \prime \prime}\right\rangle & =\frac{1}{\sqrt{2}}(\sqrt{2} \alpha b|0\rangle+\beta a|1\rangle)_{3}|11\rangle_{24}
\end{aligned}
$$




$$
\left|\zeta_{4}^{\prime \prime \prime}\right\rangle=\frac{1}{\sqrt{2}}(\sqrt{2} \alpha b|0\rangle-\beta a|1\rangle)_{3}|11\rangle_{24}
$$

Now the state of the particle 3 can be denoted as follows, respectively

$$
\begin{aligned}
\left|\varphi_{1}^{\prime \prime}\right\rangle & =\frac{1}{\sqrt{2}}(\alpha a|1\rangle+\sqrt{2} \beta b|0\rangle)_{3} \\
\left|\varphi_{2}^{\prime \prime}\right\rangle & =\frac{1}{\sqrt{2}}(\alpha a|1\rangle-\sqrt{2} \beta b|0\rangle)_{3} \\
\left|\varphi_{3}^{\prime \prime}\right\rangle & =\frac{1}{\sqrt{2}}(\sqrt{2} \alpha b|0\rangle+\beta a|1\rangle)_{3} \\
\left|\varphi_{4}^{\prime \prime}\right\rangle & =\frac{1}{\sqrt{2}}(\sqrt{2} \alpha b|0\rangle-\beta a|1\rangle)_{3}
\end{aligned}
$$

Thirdly, in order to get the unknown particles, Charlie has to introduce an auxiliary particle $|0\rangle_{g}$. From the above state of particle 3 , we can see, if the state of the particle 3 is $\left|\varphi_{1}^{\prime \prime}\right\rangle$ or $\left|\varphi_{2}^{\prime \prime}\right\rangle$, Charlie first performs the unitary measurement $U_{1}$ on particle 3 and the auxiliary particle, then performs Von Neumann measurement on the auxiliary particle. Similarly, the teleportation is succeed with the probability $\frac{a^{2}}{2}$

If the state of the particle 3 is $\left|\varphi_{3}^{\prime \prime}\right\rangle$ or $\left|\varphi_{4}^{\prime \prime}\right\rangle$, Charlie first performs the unitary measurement $V_{1}$ on particle 3 and the auxiliary particle, then performs Von Neumann measurement on the auxiliary particle. Similarly, the teleportation is succeed with the probability $\frac{a^{2}}{2}$.

So the total probability of the successful teleportation is $\frac{a^{2}}{2} \times 4=2 a^{2}$.

\section{Conclusion}

This paper improves the four-particle asymmetric entangled state channel in [10], enlarging the range of its coefficients. And we analyze the teleportation in three situations, one of David, Bob and Charlie is the receiver, and the other is the controller. It is found that the maximum probability of quantum teleportation in each case is $2 \mathrm{a} 2$. That is, although the recipients are different, the maximum probability of successful quantum teleportation is equal. The scheme can be generalized to multi-particle controlled quantum teleportation.

\section{References}

[1]. C. H. Bennett, G. Brassard, C. Crepeau, et al. Teleporting an Unknown Quantum State viaDual Classical and Einstein-PodolskyRosen Channels[J]. Phys. Rev. Lett, 1993, 70(13): 1895-1899.

[2]. X. Q. Su, G. C. Guo. Quantum Teleportation[J]. Progress in Physics, 2004, 24(3): 259-274.

[3]. H. Tang, J. X. Fang, X. M. Qian. Entanglement Teleportation in Two Quantum Channels[J]. Journal of Jiangsu Teachers University of Technology, 2006, 12(6): 15-20.

[4]. L. B. Chen, R. B. Jin. Realization of Two-destination Teleportation by Using Two Partially Entangled States[J]. Journal of Foshan University(Natural Science Edition), 2008, 26(2): 1-4.

[5]. L. Ye, G. C. Guo. Probabilistic Teleportation of an Unknown Atomic State [J]. Chinese Physics. 2002, 11(10): 996-999.

[6]. X. M. Xiu, L. Dong, Y. J. Gao. Probabilistic Teleportation of an Unknown One-Particle State by a General W State[J]. Commun. Theor. Phys, 2007, 47(3): 625C628.

[7]. H. Liu, F. L. Yan. Teleportation of an Unknown Single-particle State[J]. Journal of Hebei Normal University(Naturnal Science Edition), 2007, 31(3): 321-323.

[8]. X. S. Xiong, J. Fu, K. Shen. Teleportation of an Unknown Quantum State Under the Control of Mulltiuser[J]. Journal of Changchun University of Science and Technology, 2006, 29(3):8-11.

[9]. Z. H. Hong, Y. Y. Xia, Y. B. Huang, H. S. Li, X. J. Yi. Controlled Quantum Teleportation via Four Particle Cluster State[J]. Chinese Journal of Quantum Electronics, 2008, 25(4): 458-461.

[10]. J. Z. Zhang, W. H. Zhang. Two Quantum Secret Sharing Scheme Based on Four-qubit Entangled States[J]. Application Research of Computers, 2016, 33(1): 225-228.

[11]. K. Liu, Y. H. Li, Z. T. Liang, J. C. Liu, Y. Y. Nie. Controlled Quantum Teleportation via SixQubit Cluster State[J]. Journal of Jiangxi Normal University(Naturnal Science Edition), 2010, 34(6): 612- 615.

[12]. J. Q. Sha, J. X. Fang, W. X. Jing. Quantum Teleportation of One-to-Many Using (n+1)-Particle Entanglement[J]. Commun. Theor. Phys. (BejingChina), 2007, 48(4): 623-625. 